\title{
Roger Botte, Esclavages et abolitions en terres d'islam
}

André Versaille, 2010, 389 p.

Jean-Louis Triaud

\section{OpenEdition}

\section{Journals}

Édition électronique

URL : http://journals.openedition.org/assr/22879

DOI : $10.4000 /$ assr. 22879

ISSN : $1777-5825$

Éditeur

Éditions de l'EHESS

Édition imprimée

Date de publication : 31 décembre 2011

Pagination : 124

ISBN : 9782713223273

ISSN : 0335-5985

\section{Référence électronique}

Jean-Louis Triaud, «Roger Botte, Esclavages et abolitions en terres d'islam », Archives de sciences sociales des religions [En ligne], 156 | octobre-décembre 2011, document 156-25, mis en ligne le 14 février 2012, consulté le 21 septembre 2020. URL : http://journals.openedition.org/assr/22879 ; DOI https://doi.org/10.4000/assr.22879

Ce document a été généré automatiquement le 21 septembre 2020

(C) Archives de sciences sociales des religions 


\section{Roger Botte, Esclavages et abolitions en terres d'islam}

André Versaille, 2010, 389 p.

Jean-Louis Triaud

\section{RÉFÉRENCE}

Roger Botte, Esclavages et abolitions en terres d'islam, Bruxelles, André Versaille, 2010, $389 \mathrm{p}$.

1 Les médias ont récemment découvert ce qu'il est convenu d'appeler la "traite arabe ». Celle-ci est enseignée de longue date par les historiens, mais il est vrai qu'elle n'avait pas toujours atteint le grand public. Cette insistance mise sur la traite arabe relève souvent d'une intention polémique: stigmatiser l'islam en tant que tel, nourrir la « guerre des civilisations » et « rééquilibrer » la traite atlantique par la traite arabe, avec, parfois, une guerre des chiffres pour grossir l'une par rapport à l'autre. Il est vrai qu'en sens inverse un certain idéalisme postcolonial avait pu tendre à minorer ou occulter les formes d'esclavage pratiquées dans les pays du tiers-monde, qu'il s'agisse des pays arabo-musulmans ou africains.

2 Tout chercheur est donc aujourd'hui exposé à une instrumentalisation de la question à des fins idéologiques. C'est un des risques que devait affronter Roger Botte et qu'il a surmonté par une attention aux sources, aux représentations et aux variations du thème selon les moments et les lieux - d'où les différents pluriels contenus dans le titre. L'auteur ne s'embarque pas, pour sa part, dans la difficile guerre des chiffres, se contentant, dans son avant-propos (p. 8), d'une fourchette - de 11500000 à 17000000 de personnes africaines déportées entre 650 et 1900, toutes directions confondues (Sahara, mer Rouge et océan Indien) - qui mériterait d'ailleurs d'être accréditée par quelques références. 
3 Et c'est bien parce que, selon ses termes, «ce travail d'anthropologie et d'histoire ne défend aucune thèse » (p.7) qu'il n'en est que plus percutant sur la réalité d'une institution et sa pratique de longue durée dans le monde musulman.

Le livre est, en apparence, relativement modeste : moins de trois cents pages de texte, sans compter un lexique des termes arabes (p. 297-315) et un index (p. 379-385), tous deux également précieux. C'est, en fait, le résultat de nombreuses années d'accumulation et de travail d'analyse, en même temps qu'un monument d'érudition dans son domaine. L'anthropologue s'est fait ici islamologue et historien, puis politologue, couvrant un champ chronologique qui va des premiers siècles de l'islam aux événements les plus contemporains, adaptant à chaque fois ses outils à la nature des sources et des documents.

5 L'ouvrage est construit en six chapitres d'une quarantaine de pages chacun. «Les différents chapitres de l'ouvrage peuvent se lire indépendamment les uns des autres même si le tout constitue bien une unité»(p.9). Le premier chapitre, intitulé "L'abolition de l'esclavage au regard de la shari’a", prend à bras le corps les "fondements scripturaires de l'esclavage » et "les obstacles juridiques à l'abolition » (p.14 et 22). Il nous livre un exposé mûri sur les apories du Coran et de la tradition islamique en matière d'esclavage. Il montre bien la tension permanente, le rapport conflictuel, à partir du $\mathrm{xIX}^{\mathrm{e}}$ siècle, entre l'orthodoxie religieuse et les nécessités politiques. De là les énormes difficultés pour la pensée musulmane réformiste des XIX et $\mathrm{xx}^{\mathrm{e}}$ siècles à se frayer un chemin.

6 Faut-il y voir une tache consubstantielle à l'islam et, par conséquent, développer une vision essentialiste des rapports entre l'islam et l'esclavage ? Ce n'est pas la position de l'auteur qui y voit avant tout le produit des forces économiques, et, par conséquent, de l'évolution technologique et matérielle des sociétés concernées. Notons au passage que l'esclavage tardif n'est pas une exclusivité du monde musulman. Il ne fut aboli officiellement en Chine qu'en 1910 et ne disparut pas aussitôt. Quant à l'Afrique, qui occupe une place privilégiée dans cet ouvrage rédigé par un anthropologue de l'Afrique subsaharienne, il est bon de rappeler l'existence généralisée de l'institution servile et de traites africaines internes au sud du Sahara, y compris dans des zones comme l'Afrique centrale ou australe, qui n'avaient rien à voir avec l'islam, et dont une partie approvisionnait, sur les côtes, la traite atlantique.

7 Les textes fondateurs de l'islam, pas plus que ceux du christianisme, n'ont demandé l'abolition de l'esclavage. Ils le considéraient comme une institution «naturelle » dans les sociétés de l'époque. Les quatre Évangiles n'en parlent pas, et n'appellent pas davantage à son abolition. L'auteur rappelle opportunément (p. 278) comment Benoit XVI, dans l'encyclique Spe Salvi facti sumus (2007) tire argument d'un texte de saint Paul pour montrer que «le christianisme n'avait pas apporté un message révolutionnaire comme Spartacus » en citant à l'appui une lettre dans laquelle Paul renvoie à son ami Philémon un esclave fugitif, converti entre temps, en lui recommandant simplement de le recevoir désormais « non plus comme un esclave mais comme bien mieux qu'un esclave : un frère bien-aimé » (Épitre à Philémon, 16). Plus nettement encore, dans une autre Épitre : «Serviteurs, obéissez à vos maîtres selon la chair avec crainte et tremblement, dans la simplicité de votre cœur, comme au Christ (...) Servez-les de bon gré comme si vous serviez le Seigneur et non les hommes (...) Quant à vous, maitres, agissez de même à l'égard de vos serviteurs; abstenez-vous de 
menaces, sachant que leur Maître et le vôtre est dans les cieux et que devant lui il n'y a pas de considération de personnes » (Épitre aux Éphésiens, 5-9).

8 Sur des bases scripturaires analogues (le caractère naturel, donc voulu par Dieu, de la différenciation sociale et de l'esclavage), les évolutions dans le temps ont ensuite été différentes. Ce ne sont pas les «textes fondateurs", quand bien même les lettres de Paul ne sont pas réputées, comme le Coran, paroles divines, qui ont modifié la perception de l'esclavage, mais les conditions agricoles, économiques et sociales, et l'évolution des sociétés elles-mêmes. Il ne s'agit donc pas, pour l'auteur, de faire une explication de l'esclavage "par l'islam », qui plus est un islam essentialisé, mais, ce qui est différent, d'expliquer comment les sociétés musulmanes historiques ont eu du mal à se débarrasser d'une institution qui avait pris place, puis perduré, dans les codes juridiques constitués aux premiers siècles de l'Hégire. Cette situation renvoie, d'une façon plus globale, à l'obstacle que constituent, dans l'effort de passage des sociétés musulmanes à la modernité, non pas tant les textes sacrés initiaux que l'appareil juridique élaboré dans les premiers siècles de l'islam. Là est le cœur de la réflexion de l'auteur.

Dans le chapitre 1, il montre bien comment l'absence de condamnation explicite de l'esclavage dans le Coran contribue à figer une législation anachronique et oblige les réformistes de l'islam à jongler avec les textes, en élargissant, par exemple, les conseils qui y sont donnés de bien traiter les esclaves et de les affranchir à titre personnel pour faire une action pie, ou en défendant le principe selon lequel seul un djihad régulièrement proclamé pourrait autoriser une réduction licite (des vaincus) en esclavage.

Les chapitres suivants sont consacrés à des études de cas solidement documentées, d'où ressort bien l'affrontement entre les pensées traditionalistes, attachées à un corpus juridique rigide et fixiste, et les recours, par des intellectuels, hommes de religion ou de pouvoir, au XIX ${ }^{\mathrm{e}}$ siècle, à des arguments et interprétations modernistes. On voit aussi comment, dans ces différentes sociétés marquées par le traditionalisme religieux, ce sont "l'ingérence juridique» de la colonisation (p. 23), l'environnement et les pressions du monde occidental, qui entraînent les décisions d'abolition. Les intérêts économiques et politiques obligent partout les dirigeants musulmans à se conformer aux normes occidentales et à les légitimer.

11 L'auteur a étudié cinq pays sensibles: la Tunisie, l'Arabie saoudite, le Maroc, la Mauritanie et le Soudan. Dans chacun de ces pays, il montre comment le statut juridique servile s'accommode de situations sociales extrêmement variées, depuis celles des domestiques et concubines dans les maisons des particuliers jusqu'à celles des esclaves de cour, souvent en charge de positions stratégiques et de confiance - l'intérêt d'un esclave étant, pour le souverain, qu'il n'appartient pas à un lignage. Partout aussi, les esclaves blancs (mamlúk), originaires de pays de la Méditerranée et de la mer Noire, jouissent d'un statut supérieur à celui des Noirs - lesquels sont victimes de la malédiction biblique de Hâm (Cham), maudit par son père Noé (Nouh dans le Coran), pour lui avoir manqué de respect (Genèse, IX, 21-27 : c'est d'ailleurs, en fait, le fils de Ham, Canaan, qui est condamné à devenir « l'esclave des esclaves » de ses oncles). Là où le texte biblique ne parlait pas de couleur de peau, un mythe s'est développé, aussi bien du côté chrétien que musulman, faisant de la noirceur la marque du châtiment et, de ce fait, la justification de l'état servile des Noirs, considérés comme les descendants de Hâm. Le Coran ne reprend pourtant pas ce récit mais parle seulement de la malédiction 
d'un « fils » de Nouh qui a refusé de monter dans l'Arche (XI, 40-46) et dont le nom n'est pas mentionné mais que la tradition islamique identifie à Canaan. Le mythe double ainsi les textes canoniques.

Dans chacun des pays étudiés, la question de l'abolition de l'esclavage évolue à la fois en fonction des contraintes extérieures et des conditions locales. En Tunisie, comme en Algérie et au Maroc, des Maisons confrériques réunissaient les esclaves noirs autour de rituels communautaires regardés avec suspicion par les autorités religieuses. C'étaient des lieux de solidarité, où l'on célébrait des cultes aux génies et des danses de possession. C'étaient, aussi, des lieux porteurs d'identité - réelle ou adoptée - puisque chaque Maison portait le nom d'une ethnie subsaharienne. Sîdî Bilâl (Blal), l'esclave abyssin du Prophète et premier muezzin de l'islam, était la figure tutélaire de ces groupements et leur donnait une caution d'islamité (p. 68). Dans les années 1840, le bey de Tunis, sous la pression des consuls européens, notamment britanniques, prononça successivement l'interdiction d'exportation, la suppression du marché spécialisé et, enfin, l'émancipation des esclaves dans toute la Régence. Face à l'opposition des milieux traditionalistes et des marchands, le bey trouva appui sur les autorités religieuses officielles de l'époque, et aussi sur l'élite blanche d'origine servile, d'où étaient issus nombre de Premiers ministres de la Régence au XIXe siècle (p. 63). Ce fut avant tout une décision d'État, qui n'entraîna aucun courant d'opinion en faveur de l'abolition.

13 L'Arabie saoudite représente un autre cas de figure. La Mecque était, en particulier, un marché d'esclaves important et l'auteur souligne «le lien séculaire entre pèlerinage, traite des esclaves et leur redistribution dans l'ensemble du monde musulman » (p. 10). Dans ce berceau du littéralisme et du fondamentalisme wahhabites, où la famille royale possédait environ $60 \%$ des esclaves, la plupart noirs (p. 106), la monarchie a dû s'incliner devant les réalités économiques et politiques. La modification des rapports sociaux induite par le développement de l'industrie pétrolière et les effets de la crise de 1929 (effondrement de l'industrie du pèlerinage et de celle des perles), puis la forte concurrence de l'idéologie nassérienne, et son écho jusque dans la famille royale (sécession de princes libéraux), jointes aux pressions internationales, entrainent l'abolition de l'esclavage par le futur roi Faysal, alors président du conseil des ministres, en 1962 (une génération après une première interdiction du commerce des esclaves dans le royaume prononcée en 1936). C'est l'implantation du capitalisme mondial, notamment de l'industrie pétrolière, qui met fin à l'institution servile: les nouveaux besoins économiques exigent une main-d'œuvre salariée. L'interprétation des textes vient, au fur et à mesure, au secours des nécessités politiques. Ibn Sa'ud, puis Faysal, imposent leurs volontés aux 'ulamā'. On recourt au concept commode de maslaha (l'intérêt général) pour passer outre, de façon encore plus aisée qu'en Tunisie, aux résistances des traditionalistes. Cette synthèse de la question de l'esclavage en Arabie, rendue par l'auteur dans toute sa complexité, est assurément un modèle du genre dont nous ne pouvons ici qu'évoquer les grands traits.

À l'encontre de ce que nous pourrions penser, l'affaire se révèle plus difficile au Maroc : « cas remarquable de résistance de l'institution servile, le Maroc est, en effet, le seul pays en terres d'islam où l'esclavage ne fut jamais formellement aboli » (p. 10 et 145). C'est implicitement, en proclamant tous les Marocains égaux, que la loi fondamentale de 1961 a mis fin de facto au statut servile. Dans les années 1840, à la différence de la Tunisie, le sultan du Maroc, exposé aux mêmes représentations de la diplomatie 
britannique, répondait que l'institution servile était conforme à la Loi islamique. Quarante ans plus tard, un ministre chérifien réaffirmait encore que l'abolition serait contraire aux fondements du droit musulman (p.151). L'auteur, qui tire profit, notamment, des travaux d'an-Nâsirî (1895) et d'Ennajî (1997), consacre plus particulièrement son analyse du cas marocain à une étude des marchés d'esclaves, aux modalités de vente, à l'utilisation des esclaves dans les métiers urbains. Se profile aussi la figure de la 'ariffa, la maîtresse femme en charge des autres esclaves du Palais et du harem. L'auteur fait une trop brève digression sur les esclaves chrétiens (p. 173-175), sujet difficile qui le met aux prises avec les polémiques sur le sujet: entre les tenants d'une martyrologie chrétienne à des fins religieuses (un genre littéraire né en Europe au XvII ${ }^{\mathrm{e}}$ siècle) et ceux qui observent le sort, parfois prospère (mais parfois aussi beaucoup plus dur), des esclaves chrétiens, plus ou moins assistés par des missions franciscaines et périodiquement rachetés par les consulats. L'auteur a hésité au seuil d'un sujet un peu distinct qui l'aurait emmené trop loin.

À la différence du Maroc, qui n'a jamais proclamé l'abolition, la Mauritanie détient « le record mondial des abolitions de l'esclavage » (p. 189), soit quatre, dont une française : 1905, 1961, 1980, 2007. L'institution servile est ici très profondément inscrite dans l'ordre social à un point tel qu'elle reste une réalité actuelle, périodiquement dénoncée par les associations internationales. Il n'existe aucune statistique globale fiable du nombre des esclaves et des harātīn - cette dernière catégorie constituée d'affranchis, de culture maure comme leurs maitres -, tous massivement utilisés dans les travaux productifs et domestiques. Si l'on recoupe les quelques exemples donnés à partir de statistiques tribales des années cinquante (p. 208-209), il apparait que le total des harātin et des esclaves approche ou dépasse les $50 \%$ de la population totale, à quelques exceptions près. L'auteur, qui évoque les difficultés des anciens esclaves à vivre loin de leurs maîtres, dont le patronage reste recherché pour des raisons économiques, souligne le dilemme des harātīn, " ces exclus de la généalogie » (p. 233), partagés entre le désir de s'assimiler à l'idéologie dominante et celle de s'émanciper. Dans cette société, « où le nom détermine instantanément la place que l'on occupe au sein de la hiérarchie statutaire et sociale » (p. 235), le nom (dépréciatif) porté par un descendant d'esclave signale immédiatement le stigmate servile. L'auteur a des formules heureuses pour qualifier le statut social comme le résultat d'un " patrimoine génétique » (p. 234) et parler, à propos des esclaves, de "carence généalogique», de "violence généalogique " face aux maîtres qui se distinguent par un "capital patronymique héréditaire » (p. 236). Et l'« islam » dans tout cela? Le paradoxe est que les groupes islamistes exercent une attraction sur ces catégories. Les islamistes, qui récusent la validité du droit malékite et les hiérarchies statutaires jugées étrangères à l'islam, vont jusqu'à accepter, pour diriger la prière, des imams d'origine servile. L'injonction coranique "Tous les musulmans sont frères » (XLIX,10), dont on s'étonne qu'elle soit absente des débats du XIx ${ }^{e}$ siècle comme de l'ouvrage, est ici prise au pied de la lettre. On regrettera peut-être que, dans ce chapitre sur la Mauritanie, l'auteur ne considère que la partie (spatialement majoritaire) maure, là où les maîtres sont, en gros, «blancs» (ils se nomment d'ailleurs bìdān, "blancs») et les esclaves et harātīn, «noirs». En fait, les mêmes hiérarchies sociales règnent dans les sociétés du sud mauritanien négro-africain (vallée du fleuve Sénégal), là où maîtres et esclaves sont tous noirs. 
16 Le dernier chapitre est consacré à un pays majeur sur la scène de l'esclavage en Afrique, le Soudan. Le trafic à grande échelle d'esclaves dans cette zone remonte loin dans l'histoire. Elle a pris récemment une ampleur considérable dans les médias internationaux parce que, contrairement à la Mauritanie, il y a ici de fortes odeurs de pétrole. Comme le déplore crûment l'organisation People at Watch, "personne n'est intéressé par un pays [la Mauritanie] parlant français de seulement deux millions d'habitants et sans pétrole»(p.279). L'auteur y ajoute une dimension apparue essentielle: le Soudan est emblématique d'une situation où l'on peut présenter l'affrontement entre maîtres et esclaves comme un affrontement entre Arabes nordistes blancs et musulmans et esclaves sudistes noirs et chrétiens (ou animistes). Le Soudan devient, à ce titre, l'un des théâtres privilégiés de la " guerre des civilisations ». L'auteur montre à quel point ces représentations sont pourtant schématiques : ni le Nord, ni le Sud ne sont homogènes, y compris linguistiquement. La capture d'esclaves avait d'ailleurs lieu aussi bien dans les guerres intertribales du Sud. Quant aux Arabes soudanais, on rappellera qu'ils sont noirs de peau (p. 255) et que ce n'est donc pas leur couleur, mais leur langue (quoiqu'il y ait des langues distinctes de l'arabe au nord), leur généalogie et leur mode de vie qui les différencient. Pour autant, la capture d'esclaves du Sud par des tribus arabes du Nord est bien une réalité historique ancienne, dont l'auteur rappelle avec précision les hauts et les bas liés au contexte politique tout au long du $\mathrm{xIX}^{\mathrm{e}}$ siècle, la région du Bahr el-Ghazal constituant le nœud géographique de tous les trafics. Mais le morceau de bravoure de l'auteur porte sur l'instrumentalisation idéologique de ce conflit, au nord, par les islamistes qui instaurent la charia, puis lancent des milices tribales ravager pour leur propre compte l'ouest et le sud du Soudan, et, au sud, par les missions et les prédicateurs évangéliques qui montent des campagnes de propagande en direction des États-Unis, où leurs appels trouvent une résonance pour des raisons religieuses, humanitaires et historiques. Le succès de ces campagnes s'inscrit aussi dans le cadre de la politique étrangère des États-Unis, dont l'attitude à l'égard du régime de Khartoum oscille au gré de ses options géopolitiques. S'appuyant sur les révélations progressives faites par la presse internationale, y compris américaine, l'auteur décrit en détail l'imposture des campagnes médiatiques de rachat (redemption) de faux esclaves, menées autour des années 1998-2000, par l'organisation Christian Solidarity International (CSI) - vastes opérations de corruption qui profitent surtout aux commandants sudistes. Ainsi la mise en scène d'une prétendue lutte contre un esclavage par ailleurs réel est-elle devenue un business, en même temps qu'une " guerre de signes » (p. 295), dans la croisade " contre l'islam ».

17 C'est sur ces notes sinistres que se termine l'exposé de l'auteur qui aurait sans doute mérité une conclusion générale où, sans que soient ouverts de nouveaux chantiers, l'importance de la traite arabe sur les côtes swahilies de l'Afrique orientale aurait pu être mentionnée. Le Kenya et la Tanzanie auraient utilement leur place dans le paysage afin d'élargir, même brièvement, les exemples choisis au versant oriental du continent. Nous ne ferons cependant pas de mauvais procès à l'auteur. Celui-ci a choisi des cas significatifs à l'appui de ses démonstrations sans prétendre à l'exhaustivité. Il ne pouvait couvrir l'ensemble du champ concerné et ce n'était pas le but de cette entreprise.

18 Après avoir dit tout le bien que nous pensons de cet ouvrage magistral, nous voudrions signaler quelques inexactitudes, explicables par l'ampleur du champ couvert. L'auteur s'est intéressé, sur la base d'un article d'Ismael Musah Montana (2004), à un pamphlet 
d'Ahmad al-Tinbuktâwî, un Peul originaire de la boucle du Niger, lequel, à Tunis, en 1808, condamnait les cultes africains et recommandait au bey de maintenir les Noirs dans leur statut servile, afin d'éviter la propagation de ces formes d'idolâtrie (p. 72). Il y voit une corrélation avec la "pensée jihadiste», "partiellement sous influence wahhâbite» (p. 73), qui avait pris son essor en Afrique de l'Ouest au XIX siècle. La corrélation est pertinente mais le rattachement au wahhabisme de ce jihadisme n'est pas fondé. Les leaders de djihad au début du xix ${ }^{e}$ siècle, à Sokoto (nord Nigeria) comme dans la boucle du Niger, sont membres de la confrérie qâdiri et étrangers au wahhabisme. L'auteur a aussi établi un lien doctrinal, purement putatif, entre ce Tinbuktâwî et un réformiste algérien du siècle suivant, Tayeb El Oqbi - dont il affirme, sans citer de sources, que la campagne du FLN menée contre les cultes négro-africains au moment de l'Indépendance était «à l'instigation probable du courant religieux de Tayeb El Oqbi (1888-1960), un réformateur formé en Arabie saoudite et l'un des rares partisans du wahhâbisme en Algérie » (p. 71). Cela fait beaucoup d'hypothèses, et l'on voit comment, par glissements successifs, l'auteur fait apparaître une filière "wahhabite ", qui est tout sauf avérée. Tayyeb al-'Uqbî était, en fait, un des principaux compagnons du cheikh Ibn Bâdis, la figure tutélaire du réformisme islamique algérien (l'Association des oulémas). Ce courant, qui fut une composante du nationalisme algérien, tirait surtout son inspiration du cheikh Abduh, un Égyptien, l'un des leaders de la Salafiyya, un courant distinct du wahhabisme, partisan de la réouverture de l' ijtihād, le droit à un effort juridique renouvelé. Abduh était venu en Algérie, en 1903, et son modèle avait fortement influencé Ibn Bâdis. La littérature coloniale a souvent accusé les oulémas réformistes algériens de wahhabisme afin de les disqualifier. C'était une vision réductrice d'un courant qui, sans être ignorant du wahhabisme et sans le condamner, ne s'en réclamait pas. Bref, l'existence d'une filière wahhabite ne s'impose nullement dans cette démonstration. Les réformistes algériens, attachés à une "purification" de l'islam, n'avaient pas besoin du wahhabisme pour justifier une dénonciation de pratiques jugées par eux hétérodoxes, que ce soit celles des confréries « noires » comme de toutes les confréries en général.

Nous avons relevé d'autres inexactitudes plus ponctuelles :

L'auteur écrit : "Nulle allusion non plus [dans le Coran] à Agar, l'esclave d'Abraham et la mère de son fils Ismâ'̂̂l, pour lesquels Dieu fit jaillir miraculeusement la source de Zamzam à la Mecque pour sauver cet esclave et son fils qui se mouraient de soif (Genèse $\mathrm{XX}, 10-18)$ »(p.17). Il s'agit là d'un raccourci qui pourrait faire croire que c'est la Genèse qui parle de ce miracle. Ce n'est évidemment pas le cas. Ce miracle est rapporté par la tradition islamique (Bukhârî). Il reste vrai que le Coran ne parle pas d'Agar, ni la tradition islamique de son statut servile. En outre, la référence exacte à Agar et Ismaël dans la Genèse est XXV (et non XX) : 10-18. Dans le domaine géographique, enfin, le Wâdây n'est pas au Soudan (p. 66), mais au Tchad.

21 Nous voudrions aborder maintenant une question plus complexe. L'auteur, qui n'est pas arabisant, a fait un effort remarquable pour accéder à des sources arabes et procéder à une translittération rigoureuse des mots de la terminologie arabe. Il doit en être fortement remercié. Il s'est aidé pour ce faire de l'assistance de plusieurs collègues dont les noms figurent dans les remerciements globaux de la page 7. Il serait cependant préférable, sur un certain nombre de points, sensibles ou non, de savoir qui traduit et qui conseille. Il en est ainsi, notamment, toutes les fois que l'auteur renvoie à des textes 
en arabe. Cette situation peut être à l'origine d'imprécisions, dont nous signalons ici des exemples au passage :

p. 26, l'auteur écrit: "par ailleurs, le même terme (waladat), enfanter, mettre bas, s'utilisait en parlant des esclaves et des animaux ». En fait, la forme verbale est plutôt walada et elle s'utilise pour tous les êtres, y compris les femmes libres.

p. 184 et 186, il est question d'« une zawāya (sanctuaire abritant la dépouille d'un saint) ». En fait, zawāya est la forme du pluriel, et il faut lire le singulier zāwiya. Une $z \bar{a}$ wiya peut, ou non, abriter le tombeau d'un saint. Elle est le siège local d'une confrérie, avec des facilités d'enseignement et d'hébergement.

p. 303 : « raqaba, pl. raqīq ou riqāb». C'est effectivement un nom générique pour un esclave, mais raqiq ne peut pas être le pluriel de raqaba.

le mot sadāqa (p. 314) est présenté comme " aumône légale, offrande ou don pieux ", dont une part est réservée pour aider des esclaves à s'affranchir. Il y a, en fait, confusion et redondance avec zakāt, présentée quelques lignes plus loin. La sadāqa est, elle, un don volontaire pieux.

p. 326, n. 140 : Tafsir al-Manār, n'est pas l'« exégèse du minaret ", mais l'« exégèse du phare » (l'image est celle de la diffusion de la lumière) - al-Manār étant le titre de la revue fondée en 1899 par Rashîd Ridâ.

Enfin, dans le lexique final, très riche (p. 297-302), qui est beaucoup plus qu'un lexique, où l'auteur traite de l'abondante terminologie arabe de la question, il est dommage qu'il ne cite pas ses références. Non qu'il y ait doute sur l'honnêteté de ses notices, mais il y a nécessité, en pareille matière, de pouvoir remonter aux sources de l'information. Ainsi en est-il, notamment, de son exposé savant sur le "vocabulaire coranique " de l'esclavage (p. 297-302) qui aurait exigé une bibliographie propre.

Nous voudrions, pour terminer, considérer la bibliographie finale. Celle-ci témoigne de l'énorme effort documentaire effectué par l'auteur, qui a recherché aussi bien les grands classiques que les articles d'actualité et a constitué une très riche bibliothèque de la question. Il y manque peut-être, sur les esclaves zanj d'Irak, utilisés dans les plantations de Mésopotamie, l'ouvrage connu d'Alexandre Popovic, La révolte des esclaves en Irak au III'/IXe siècle (Paris, Geuthner, 1976), que l'on attendrait à l'appui de la note 125 (p. 325), mais c'est peu de chose au regard du dépouillement effectué.

La manière dont cette bibliographie est présentée appelle un commentaire. L'auteur indique, en effet, qu'il s'agit d'une "bibliographie sélective», avec cette précision: "Toutes les références utilisées étant répertoriées dans les notes, il s'agit bien ici d'une bibliographie sélective. Une bibliographie exhaustive est consultable sur le site interactif de l'éditeur» (p. 369). Nous avons donc une bibliographie à trois niveaux : celle des notes des chapitres, celle de la fin de l'ouvrage, «sélective " (qui contient, en fait, une grande partie de celle des notes), et une troisième, " exhaustive » en ligne que nous n'avons d'ailleurs pas retrouvée. On peut ne pas être convaincu par ce système malcommode. Il est utile, par contre, de signaler que l'on trouve, sur le site de l'éditeur, la reproduction en PDF de 75 pages du livre, accompagnées de petits exposés en vidéo où l'auteur aborde les principaux thèmes de son ouvrage - une méthode séduisante d'utilisation d'Internet en appui à l'ouvrage.

Le relevé de ces quelques défectuosités est surtout la preuve de notre attention soutenue à la lecture d'un livre de qualité que nous voudrions aussi achevé dans les détails qu'il l'est dans sa conception et son exposition. La thèse défendue, preuves et 
exemples à l'appui, bat en brèche les théories essentialistes et culturalistes sur l'« islam ». L'auteur met en scène un univers culturel et religieux complexe, traversé par des débats et marqué par la pluralité des pratiques et des adaptations, des croisements aussi, comme en Mauritanie, avec des héritages culturels locaux. La référence, dans le titre, à des terres plurielles et non à un islam générique marque toute la différence. Tout au long de cet exposé, la question posée n'est pas tant celle de savoir " ce que dit l'islam » sur l'esclavage, mais ce que différents acteurs, en différents lieux et en des moments différents, lui font dire. Roger Botte a fait preuve, à cette occasion, d'une grande maîtrise des appareils idéologiques comme des situations sociales et des histoires régionales. C'est un ouvrage que l'on peut lire d'un seul trait ou à petits pas, mais qui constitue désormais une ressource indispensable. 